\title{
Microtubule Dynamics Depart from the Wormlike Chain Model
}

\author{
Katja M. Taute, ${ }^{1}$ Francesco Pampaloni, ${ }^{2}$ Erwin Frey, ${ }^{3}$ and Ernst-Ludwig Florin ${ }^{1, *}$ \\ ${ }^{1}$ Center for Nonlinear Dynamics, University of Texas at Austin, 1 University Station C1610, Austin Texas 78712, USA \\ ${ }^{2}$ Cell Biology and Biophysics Unit, European Molecular Biology Laboratory, Meyerhofstraße 1, 69117 Heidelberg, Germany \\ ${ }^{3}$ Arnold Sommerfeld Center for Theoretical Physics and CeNS, Department of Physics, Ludwig-Maximilians-Universität München, \\ Theresienstraße 37, D-80333 München, Germany \\ (Received 11 September 2007; published 15 January 2008)
}

\begin{abstract}
Thermal shape fluctuations of grafted microtubules were studied using high resolution particle tracking of attached fluorescent beads. First mode relaxation times were extracted from the mean square displacement in the transverse coordinate. For microtubules shorter than $\sim 10 \mu \mathrm{m}$, the relaxation times were found to follow an $L^{2}$ dependence instead of $L^{4}$ as expected from the standard wormlike chain model. This length dependence is shown to result from a complex length dependence of the bending stiffness which can be understood as a result of the molecular architecture of microtubules. For microtubules shorter than $\sim 5 \mu \mathrm{m}$, high drag coefficients indicate contributions from internal friction to the fluctuation dynamics.
\end{abstract}

DOI: 10.1103/PhysRevLett.100.028102

PACS numbers: 87.16.Ka, 87.15.La

Microtubules are cytoskeletal protein filaments that play an essential role in a multitude of cell functions in all eucaryotes. While specialized structures such as cilia, flagella, and axons require microtubule lengths of up to several hundred micrometers, fundamental tasks like cell division and intracellular transport involve microtubules with lengths that are comparable to or smaller than typical eucaryotic cell sizes $(10-20 \mu \mathrm{m})$. In solution, microtubules undergo strong thermal shape fluctuations but, due to their rigidity, maintain an average direction and are therefore referred to as semiflexible. The standard model for semiflexible polymers is the wormlike chain $[1,2]$ which envisions a homogeneous, isotropic, continuously flexible rod characterized by its bending stiffness, $\kappa$.

Recently, evidence has accumulated suggesting that the wormlike chain as the standard model for semiflexible polymers may constitute an oversimplification in the case of microtubules due to their highly anisotropic molecular architecture [3,4]. Microtubules consist of strings of $\alpha$ - and $\beta$-tubulin heterodimers, so-called protofilaments, that are arranged in parallel forming a hollow tube of $25 \mathrm{~nm}$ external diameter. Neighboring protofilaments are shifted relative to each other, giving rise to helicity. Both the rise per turn and the number of protofilaments may vary; however, 3 monomers per turn and 13 constituent filaments are most common in vivo [5,6]. This protofilament architecture makes microtubules a model system for the generalized theory of wormlike bundles [7,8], which in addition to various other biological examples also describes the mechanics of carbon nanotube bundles.

Few studies have addressed the dynamics of thermal shape fluctuations of microtubules even though the time scales of these fluctuations affect the time scales on which biological functions occur. Caspi et al. [9] examined the transverse mean square displacement of microtubules in networks, finding a power law behavior $t^{3 / 4}$. Janson and
Dogterom [10] extracted autocorrelation times for several modes from a shape analysis of growing grafted microtubules, while Brangwynne et al. [11] applied this procedure to stabilized fluorescent microtubules. For technical reasons, all of these studies restricted themselves to lengths of several tens of micrometers, while microtubule lengths relevant to cell functions such as cell division are much shorter. In a study of equilibrium position distributions [4], the most significant systematic deviations from the wormlike chain model were found for microtubules shorter than $\sim 20 \mu \mathrm{m}$ which coincides with the length regime most crucial to cell division and intracellular transport. This suggests that, in this length regime, corresponding deviations should exist for dynamical parameters such as the relaxation time.

In this Letter, we present an analysis of first mode relaxation times for microtubules of length $2-30 \mu \mathrm{m}$. Microtubules are grafted to a substrate at one end and data are extracted from the mean square displacement of the transverse coordinate. Capturing dynamics for microtubules that are only several $\mu \mathrm{m}$ long is challenging since the corresponding spatial and temporal scales become increasingly small. We overcome these difficulties by using small fluorescent beads attached to the microtubules as tracer particles. Their position can be tracked at frame rates of up to $30 \mathrm{~Hz}$ using a high quantum efficiency CCD camera. In contrast to shape analysis techniques that provide low resolution (tens of nanometers $[10,11]$ ) position data for the whole filament, the use of the tracer bead yields spatial information with a precision of a few nanometers for one specific point on the filament's contour. Standard semiflexible polymer models are then used to infer relaxation times, bending stiffnesses, and drag coefficients.

In the wormlike chain model, relaxation times for a filament are given by [12] 


$$
\tau_{n}=\frac{\zeta L^{4}}{\kappa q_{n}^{4}}
$$

where $\zeta, L$, and $\kappa$ denote the drag coefficient per unit length, the filament's contour length, and its bending stiffness, respectively, while $q_{n}$ is a mode-dependent numerical factor which corresponds to a wave number normalized by length and is determined by the boundary conditions. An estimate of $\zeta$ can be obtained from the formula for a rigid cylinder of length $L$ and diameter $d$ held steady in a homogeneous flow of a fluid of viscosity $\eta$ [13] at low Reynolds number:

$$
\zeta_{0}=\frac{4 \pi \eta}{\ln (L / d)+2 \ln (2)-1 / 2} .
$$

The Stokes friction coefficient for the attached bead is $\gamma_{\text {bead }}=6 \pi \eta r \approx 1.9 \times 10^{-9} \mathrm{Ns} / \mathrm{m}$ for $\eta \approx 10^{-3} \mathrm{~N} \mathrm{~s} / \mathrm{m}^{2}$ and $r=100 \mathrm{~nm}$ being the viscosity of water and the radius of the bead, respectively. The drag $\zeta_{0} L$ acting on the microtubule is significantly larger than $\gamma_{\text {bead }}$ and only becomes comparable for microtubules shorter than $\sim 1 \mu \mathrm{m}$ [14]. Hence, in our case, the attached bead does not need to be considered in the analysis of the microtubule dynamics.

Because of confinement of the filament's position, the mean square displacement of the transverse coordinate saturates with time $[15,16]$ :

$$
\operatorname{MSD}(t) \propto \sum_{n} \frac{L^{3}}{\kappa q_{n}^{4}}\left(1-e^{-t / \tau_{n}}\right) f_{n}\left(L_{\mathrm{a}} / L\right),
$$

where $L_{\mathrm{a}}$ refers to the contour length from the microtubule attachment point to the bead and $f_{n}\left(L_{\mathrm{a}} / L\right)$ accounts for a modulation of the amplitude due to the position on the contour. In the case of one free and one clamped end, $q_{n}=$ $(n-1 / 2) \pi$, and the sum is taken over all modes. As $\left(q_{2} / q_{1}\right)^{4} \approx 80$, it follows that the amplitude of the first mode dominates the mean square displacement. Furthermore, Eq. (1) shows that relaxation times decrease rapidly with mode number. For fluorescence microscopy studies that rely on integration times on the order of several to tens of milliseconds, this implies that higher modes are generally averaged over during imaging and therefore do not contribute significantly to the measured MSD. Given the stiffness of microtubules, we can therefore safely assume that our measurements essentially reflect the behavior of the first mode. Contributions from the second or higher modes may occur during measurements on long microtubules where relaxation times are significantly longer; however, these are a negligible source of error compared to the statistical uncertainties in this length regime.

Microtubules were polymerized from unlabeled, rhodamine-labeled, and biotinilated tubulin (Cytoskeleton Inc, Denver, CO) and stabilized in $20 \mu \mathrm{M}$ taxol (T1912, Sigma, St. Louis, MO) to ensure stability of the length. Using standard thiol chemistry (see Ref. [4]), microtubules were covalently grafted to a microstructured gold substrate at one end, leaving the other end free to fluctuate in three dimensions. A yellow-green fluorescent bead was tightly bound to the microtubule using specific binding between avidin and biotin. A bead diameter of $200 \mathrm{~nm}$ was chosen to balance requirements on negligible drag and sufficient fluorescence intensity for high precision position tracking. Figure 1 shows a schematic of the assembly. For 20 microtubules of lengths $L=2.2$ to $27.9 \mu \mathrm{m}, 10000$ to 15000 frames of the bead's motion were taken at frame rates of 10 to $30 \mathrm{~Hz}$ using integration times of 18 to $80 \mathrm{~ms}$. Twodimensional position data were obtained from the time series using a custom-written Matlab (The MathWorks, Natick, MA) single particle tracking algorithm involving Gaussian fits to the bead's image.

MSD data obtained from time averages of finite time traces are subject to statistical errors that are nontrivial to detect and quantify. In order to avoid biased results arising from correlations in the data, error estimates on MSD data were obtained as outlined in Ref. [17].

In addition to statistical errors, instrumentation limitations need to be considered. Finite integration times average position fluctuations and thereby result in effective low-pass filtering [18]. We account for the low-pass effect by extracting relaxation times from a fit to a corrected expression for the measured MSD of first mode fluctuations [19].

In Fig. 2, the resulting relaxation time data are plotted versus the contour length [20]. For short filaments, the data approach a power law $\tau \propto L^{2}$ which differs considerably from the $\tau \propto L^{4}$ dependence predicted by the wormlike chain model. This observation leads to the conclusion that either the bending stiffness $\kappa$ or the drag coefficient $\zeta$ in Eq. (1) must have a hidden dependence on $L$.

The persistence length $l_{\mathrm{p}}=\kappa /\left(k_{B} T\right)$ can be determined from the transverse position variance $V$ using [4,21]

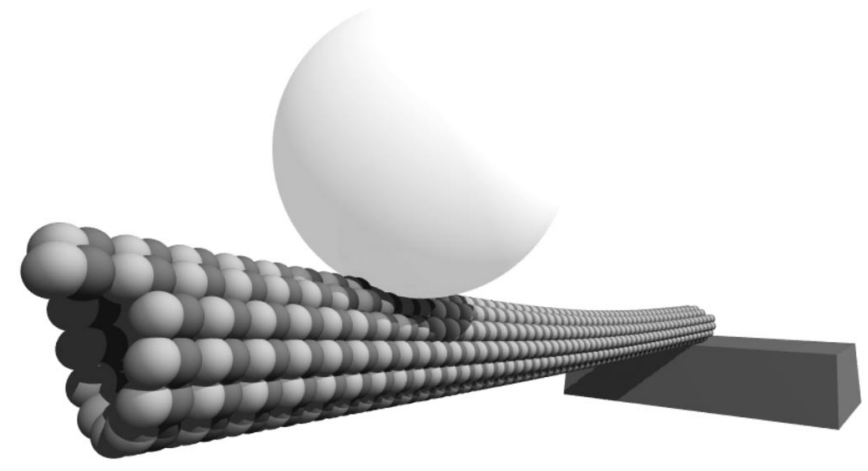

FIG. 1. Schematic of the assay. One end of the microtubule is covalently grafted on the gold substrate. Lengths are measured along the contour starting at the substrate, with $L$ denoting the free end and $L_{\mathrm{a}} \leq L$ marking the position of the tracer bead. Different fluorescent labels for the microtubule and the bead allow for separate imaging of the two and prevent photobleaching. 


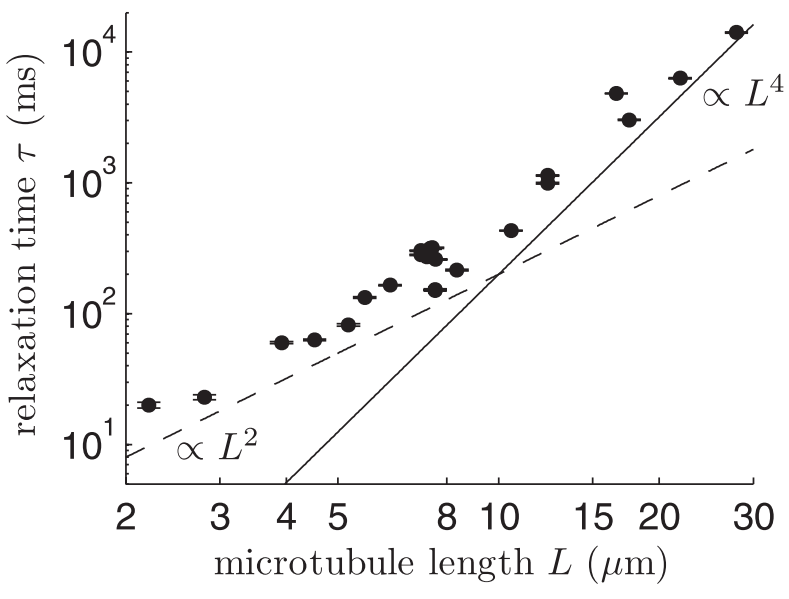

FIG. 2. Relaxation times extracted from MSD. Lines of slope 4 (solid line) and 2 (dashed line) are shown for comparison.

$$
V=\frac{L_{\mathrm{a}}^{3}}{3 l_{\mathrm{p}}}
$$

where a low-pass filtering corrected expression is applied [18]. Once $l_{\mathrm{p}}$ and hence $\kappa$ is known, the drag coefficient $\zeta$ can be computed using Eq. (1).

The plots of $l_{\mathrm{p}}$ and $\zeta$ versus $L$ in Figs. 3 and 4 immediately reveal that both quantities exhibit unexpected behavior. The persistence length shows a significant increase with overall filament length, similar to the length dependence obtained in Ref. [4] where, based on the Timoshenko beam formalism [22] that describes macroscopic elastic beams with shear contributions, the expression

$$
l_{\mathrm{p}}=\frac{l_{\mathrm{p}}^{\infty}}{1+\left(\frac{\lambda}{L}\right)^{2}}
$$

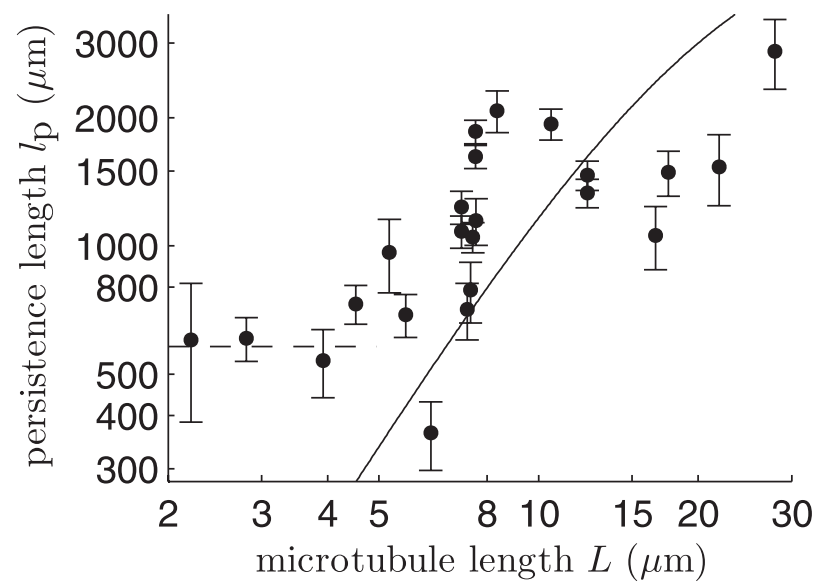

FIG. 3. Persistence lengths obtained from the position variance. A plot of Eq. (5) using the parameters of $\lambda=21 \mu \mathrm{m}$ and $l_{\mathrm{p}}^{\infty}=6300 \mu \mathrm{m}$ obtained by Ref. [4] is shown for comparison (solid line). For short microtubules, the data deviate from the Timoshenko theory and level to a plateau (dashed line). was derived. The parameter $\lambda$ accommodates for decreases in microtubule stiffness due to shear contributions from inter-protofilament sliding. In our case, however, a fit of Eq. (5) cannot provide much information as our data range does not include the saturation at $l_{\mathrm{p}}^{\infty}$ predicted for very long microtubules. While, for microtubules longer than $\sim 5 \mu \mathrm{m}$, qualitative agreement of the data with the quadratically increasing regime of Eq. (5) is evident, shorter microtubules seem to deviate from this behavior and level to a plateau. Averaging the values for the three shortest microtubules yields a value of $580 \pm 100 \mu \mathrm{m}$. Interestingly, the existence of such a plateau value is predicted by the more general theory of wormlike bundles $[7,8]$ that takes into account the microscopic filament architecture. The Timoshenko theory derives an effective bending rigidity from stretch and shear terms, which result in fully coupled bending for long microtubules and an intermediate, shear-dominated length regime where rigidity scales with $L^{2}$. In addition to these terms, the constituent filaments in a wormlike bundle have a bending stiffness that dominates the rigidity of the decoupled bundle in the short length regime.

At the same time, the drag coefficients shown in Fig. 4 significantly deviate from the hydrodynamic estimate of Eq. (2) for short microtubules. The sharp increase in this length regime is consistent with the presence of internal friction as introduced by Poirier and Marko [23], who derived an additional friction term of

$$
\zeta_{\text {int }}=\varepsilon\left(\frac{q_{n}}{L}\right)^{4}
$$

to accommodate for dissipation during conformational changes or due to liquid flowing through narrow pores in a biofilament. In the case of microtubules, both contributions seem reasonable due to the complex composite ar-

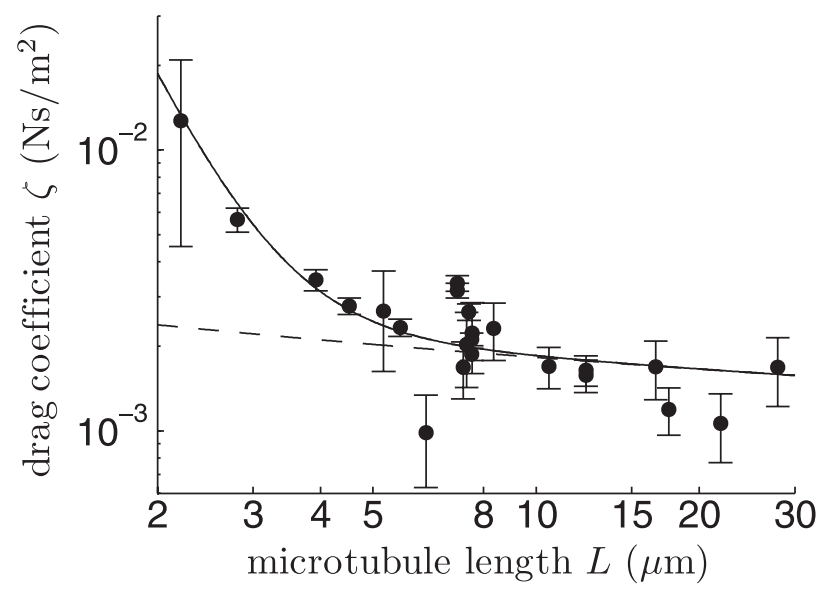

FIG. 4. Drag coefficients $\zeta$ versus contour length $L$. For short microtubules, the experimental data lie significantly above the hydrodynamic estimate $\zeta_{0}$ of Eq. (2) (dashed line). The solid line shows a fit of Eq. (7) taking into account internal friction. 
chitecture and the large fluid cylinder inside the filament. The parameter $\varepsilon$ is proportional to an effective viscosity summarizing the friction losses inside the filament. A fit of the expression

$$
\zeta=\zeta_{0}+\zeta_{\text {int }}=\frac{4 \pi \eta}{\ln (L / d)+2 \ln (2)-1 / 2}+\varepsilon\left(\frac{q_{1}}{L}\right)^{4}
$$

with $q_{1}=\pi / 2$ yields a coefficient $\varepsilon=4.3 \pm 0.5 \times$ $10^{-26} \mathrm{~N} \mathrm{~s} \mathrm{~m}^{2}$ which is more than 1 order of magnitude smaller than the numbers of $1.6 \times 10^{-24} \mathrm{~N} \mathrm{~s} \mathrm{~m}^{2}$ and $6.9 \times$ $10^{-25} \mathrm{~N} \mathrm{~s} \mathrm{~m}^{2}$ obtained by Brangwynne et al. [11] and Janson and Dogterom [10], respectively. This discrepancy suggests that high mode deformations of long microtubules may be governed by different dissipative mechanisms than first mode deformations of short microtubules.

In this Letter, we presented a first analysis of relaxation times and drag coefficients for microtubules in the length range most relevant to essential cellular functions such as cell division. Our results show that the dynamic behavior for this short length regime significantly deviates from predictions of the wormlike chain model. The dynamics seem to be modulated by a length-dependent persistence length as well as additional friction contributions that may result from internal dissipation. While microtubules longer than $\sim 5 \mu \mathrm{m}$ display a persistence length behavior consistent with the Timoshenko model, shorter microtubules show a uniform persistence length of $580 \pm 100 \mu \mathrm{m}$ which is consistent with decoupled bending of constituent protofilaments as predicted by the theory of wormlike bundles $[7,8]$. These findings not only emphasize the importance of molecular architecture to be taken into account for models of semiflexible polymer mechanics, but also suggest a wealth of possibilities for natural modulation of biopolymer properties in cells.

The authors would like to thank Claus Heussinger for helpful discussions. K. M. T. and F. P. gratefully acknowledge support by the German National Academic Foundation and the Landesstiftung Baden-Württemberg (Forschungsprogramm Optische Technologien), respectively. E. F. acknowledges support from the DFG through SFB 486, and from the German Excellence Initiative via the NIM program. This research was supported by NSF Grant No. CMMI-0728166.

*florin@chaos.utexas.edu

[1] O. Kratky and G. Porod, Rec. Trav. Chim. 68, 1106 (1949).
[2] N. Saitô, K. Takahashi, and Y. Yunoki, J. Phys. Soc. Jpn. 22, 219 (1967).

[3] A. Kis, S. Kasas, B. Babić, A. J. Kulik, W. Benoît, G. A. D. Briggs, C. Schönenberger, S. Catsicas, and L. Forró, Phys. Rev. Lett. 89, 248101 (2002).

[4] F. Pampaloni, G. Lattanzi, A. Jonás̆, T. Surrey, E. Frey, and E.-L. Florin, Proc. Natl. Acad. Sci. U.S.A. 103, 10248 (2006).

[5] D. Sept, J. Baker, and N. A .McCammon, Protein Sci. 12, 2257 (2003).

[6] D. Chrétien, E. Metoz, E. Verde, E. Karsenti, and R. Wade, J. Cell Biol. 117, 1031 (1992).

[7] C. Heussinger, M. Bathe, and E. Frey, Phys. Rev. Lett. 99, 048101 (2007).

[8] M. Bathe, C. Heussinger, M. Claessens, A. Bausch, and E. Frey, Biophys. J. (to be published).

[9] A. Caspi, M. Elbaum, R. Granek, A. Lachish, and D. Zbaida, Phys. Rev. Lett. 80, 1106 (1998).

[10] M. Janson and M. Dogterom, Biophys. J. 87, 2723 (2004).

[11] C. Brangwynne, G. Koenderink, E. Barry, Z. Dogic, F. MacKintosh, and D. Weitz, Biophys. J. 93, 346 (2007).

[12] S. Aragón and R. Pecora, Macromolecules 18, 1868 (1985).

[13] R. Cox, J. Fluid Mech. 44, 791 (1970).

[14] Although for our shortest microtubule with $L=2.2 \mu \mathrm{m}$ the hydrodynamic estimate $\zeta_{0} L$ is only $\sim 3$ times larger than $\gamma_{\text {bead }}$, the measured drag (see Fig. 4) is more than 1 order of magnitude larger than $\gamma_{\text {bead }}$.

[15] K. Kroy and E. Frey, Phys. Rev. E 55, 3092 (1997).

[16] R. Granek, J. Phys. II (France) 7, 1761 (1997).

[17] H. Flyvbjerg and H. Petersen, J. Chem. Phys. 91, 461 (1989).

[18] W. Wong and K. Halvorsen, Opt. Express 14, 12517 (2006).

[19] With only one mode being considered, the motion of a bead attached to a fluctuating microtubule can be modeled as that of a Brownian particle in an effective harmonic trap. We hence derive a low-pass filtering correction for dynamic data building on an approach recently developed by Wong and Halvorsen [18] in the context of optical trapping. For times $t$ larger than the integration time $W$, we find $\operatorname{MSD}(t) \propto 2 \frac{\tau^{2}}{W^{2}}\left(e^{-W / \tau}-1+\frac{W}{\tau}\right)-\left(\frac{\sinh \left(\frac{W}{W}\right)}{\frac{W}{W}}\right)^{2} e^{-t / \tau}$.

[20] The large scatter in the data is due to naturat heterogeneity in microtubule mechanical properties, most likely caused by the heterogeneity in microtubule architecture.

[21] A. Gholami, J. Wilhelm, and E. Frey, Phys. Rev. E 74, 041803 (2006).

[22] J. Gere and S. Timoshenko, Mechanics of Materials (PWS-KENT Publishing Company, Boston, 1990), p. 694, 3rd ed..

[23] M. G. Poirier and J. F. Marko, Phys. Rev. Lett. 88, 228103 (2002). 